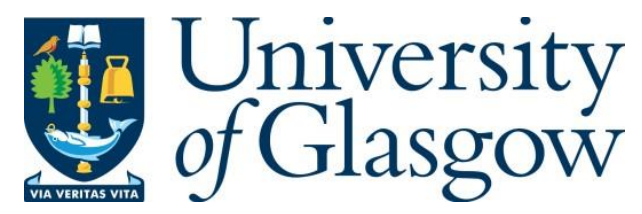

Freeman, E. , Marzo, A., Kourtelos, P. B., Williamson, J. R. and Brewster, S. (2019) Enhancing Physical Objects with Actuated Levitating Particles. In: 8th ACM International Symposium on Pervasive Displays (PerDis '19), Palermo, Italy, 12-14 Jun 2019, ISBN 9781450367516 (doi:10.1145/3321335.3324939).

There may be differences between this version and the published version. You are advised to consult the publisher's version if you wish to cite from it.

(C) The Authors 2019. This is the author's version of the work. It is posted here for your personal use. Not for redistribution. The definitive Version of Record was published in Proceedings of the 8th ACM International Symposium on Pervasive Displays (PerDis '19), Palermo, Italy, 12-14 Jun 2019, ISBN 9781450367516.

http://eprints.gla.ac.uk/185031/

Deposited on: 23 April 2019

Enlighten - Research publications by members of the University of Glasgow http://eprints.gla.ac.uk 


\section{Enhancing Physical Objects with Actuated Levitating Particles}

\author{
Euan Freeman \\ University of Glasgow \\ Glasgow, United Kingdom \\ euan.freeman@glasgow.ac.uk
}

\author{
Asier Marzo \\ Universidad Publica de Navarra \\ Pamplona, Spain \\ asier.marzo@unavarra.es
}

\author{
Praxitelis B. Kourtelos \\ University of Glasgow \\ Glasgow, United Kingdom \\ p.kourtelos.1@research.gla.ac.uk
}

Julie R. Williamson

University of Glasgow

Glasgow, United Kingdom

julie.williamson@glasgow.ac.uk
Stephen Brewster

University of Glasgow

Glasgow, United Kingdom

stephen.brewster@glasgow.ac.uk
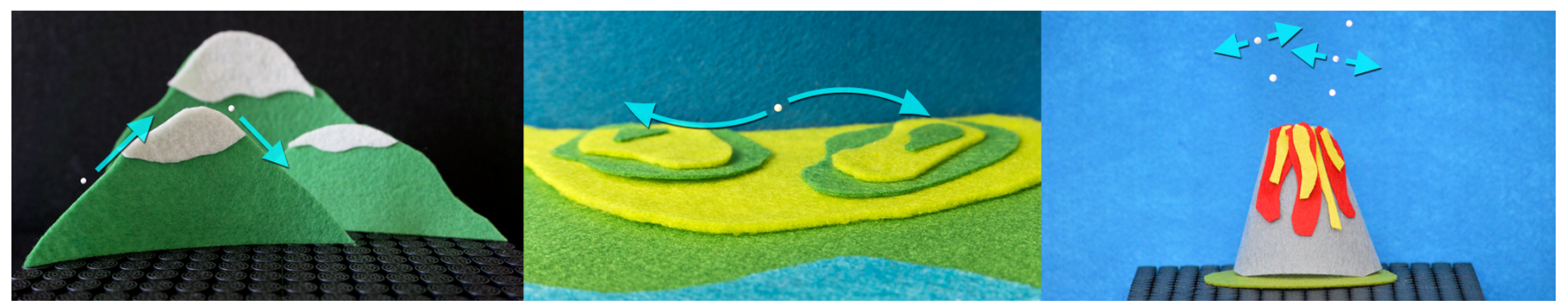

Figure 1: Levitating particles can be used to enable otherwise static objects to present dynamic visual content. These examples show physical models with polystyrene beads that are actuated by an acoustic levitation system.

\begin{abstract}
We describe a novel display concept where levitating particles are used to add a dynamic display element to static physical objects. The particles are actuated using ultrasound, for expressive output without mechanical constraints. We explore novel ways of using particles to add dynamic output to other objects, for new interactive experiences. We also discuss the practical challenges of combining these. This work shows how the unique capabilities of levitation can create novel displays by enhancing another form of media.
\end{abstract}

\section{CCS CONCEPTS}

• Human-centered computing $\rightarrow$ Interaction devices.

\section{KEYWORDS}

Acoustic Levitation, Shape-Changing Displays, Ultrasound

\section{ACM Reference Format:}

Euan Freeman, Asier Marzo, Praxitelis B. Kourtelos, Julie R. Williamson, and Stephen Brewster. 2019. Enhancing Physical Objects with Actuated Levitating Particles. In Proceedings of the 8th ACM International Symposium on Pervasive Displays (PerDis '19), June 12-14, 2019, Palermo, Italy. ACM, New York, NY, USA, 7 pages. https://doi.org/10.1145/3321335.3324939

PerDis '19, June 12-14, 2019, Palermo, Italy

(c) 2019 Copyright held by the owner/author(s). Publication rights licensed to ACM. This is the author's version of the work. It is posted here for your personal use. Not for redistribution. The definitive Version of Record was published in Proceedings of the 8th ACM International Symposium on Pervasive Displays (PerDis '19), June 12-14, 2019, Palermo, Italy, https://doi.org/10.1145/3321335.3324939.

\section{INTRODUCTION}

Acoustic levitation enables a new type of display where content is composed of small particles, levitating in air [5]. For example, a cube could be represented in-air by levitating particles located at its eight corners, or a sphere could be represented using particles arranged in a point cloud. By manipulating the sound waves in the acoustic levitator, the particles can be moved independently, in three dimensions. This allows shape-changing content without the mechanical constraints of other actuated display technologies. Users can interact inside the display volume as well, an affordance that supports a wide range of human motions and interactions with the levitating content.

Levitating particle displays do have limitations, however. The content they present is sparse, because particles need to be far enough apart (a function of the wavelength, $\lambda$ [14]). Most acoustic levitation systems use small expanded polystyrene beads $(\varnothing<5 \mathrm{~mm})$ for their display particles, because their small size $(<0.5 \lambda)$ and low density are ideal for levitating. These particles have a very simple appearance and limited expressiveness, so only their position and movements in the display can be used to convey information.

In this work, we explore an alternative way of using acoustic levitation to create dynamic displays. Instead of using particles to create sparse composite objects or point clouds, we use them to enhance the appearance of static physical objects, like the models shown in Figure 1. Levitation adds an actuated display component to these static models, turning them into dynamic, interactive displays. The simplicity of the levitating particles is not an issue when they are used alongside other forms of content, because their role is to enhance presentation and add interactivity. The context in which 
the particles appear adds meaning to their motions and enhances their expressiveness, for rich output.

We explore the novel design possibilities afforded by the combination of levitation and other materials. We investigate the ways a particle can be used to add interactivity to another item, effectively turning it into an interactive display. We then address the practical issues of this combination and present three methods for adding levitation to the space surrounding a physical object. Throughout the paper, we describe physical prototypes that use readily available devices and software platforms, to demonstrate our display concept and improve access to this technology.

\section{BACKGROUND}

\subsection{Acoustic Levitation}

The two most common acoustic levitation methods use standing waves or acoustic elements to levitate objects in air. Both are based on the principle of focusing ultrasound from one or more arrays of transducers, to create focused regions of high sound pressure. Standing wave levitation is the simplest method. Two arrays facing each other, or one array with a reflective surface placed above it, are used to create a standing ultrasound wave. Suitably sized objects $(\varnothing<0.5 \lambda)$ can be levitated in the low-pressure nodes of the standing wave. Most HCI research into levitation use this method, with two arrays of $40 \mathrm{kHz}$ transducers facing each other (e.g., [1, 4, 17, 18]).

An alternative method uses acoustic elements for levitation [15] These are shaped sound structures that can levitate and manipulate objects above a single ultrasound array. An example of an acoustic element is the 'twin trap', two adjacent areas of high sound pressure that 'pinch' an object from each side in air. Both methods can be used to levitate many objects, by creating multiple standing waves or acoustic elements. Many objects can be levitated and manipulated independently, although their density is limited by the frequency of ultrasound. Objects must be spaced far enough apart that their standing waves, or acoustic elements, do not interfere or merge [14]

Levitating objects can be actuated in mid-air by manipulating the sound field, effectively moving the standing waves [18] or acoustic elements [15]. Many objects can be moved independent of each other, a key characteristic of levitating particle displays. This capability has been used to create interactive systems based on levitation. For example, Point-and-Shake [4] animated the position of one particle as feedback, to identify which of several particles the user was interacting with. The ability to actuate levitating particles is a core component of the work described in this paper: we use particle movement as a means of adding dynamic output and interactivity to static, un-actuated physical objects.

Acoustic levitation has been used for a variety of applications in HCI research. Pixie Dust [17] was the first time acoustic levitation was used in an interactive system. They used levitating objects to form 2D graphics in a flat plane and also projected graphics onto them. The static objects were used like a 'screen' in mid-air. LeviPath [18] allowed multiple levitating objects to be moved independently along 3D paths, so objects could be used as dynamic and expressive display elements. In those systems, the object positions were used to encode information. This was also used in Floating Charts [19] for data visualisation; each levitating object represented a data point and its vertical position corresponded to its value.
New interaction techniques have been developed to allow users to manipulate the particles in a "levitating particle display" [5]. Point-and-Shake [4] used mid-air pointing gestures for object selection, a precursor to more complex actions. Users pointed a finger to select an object and the targeted object shook from side to side as feedback. LeviCursor extended the range of interactions possible with levitating objects, using in-air finger movements to directly move an object [1]. Whilst only a limited amount of feedback could be conveyed for these interactions through object movement, it was enough to support successful interaction. In this paper, we draw inspiration from these techniques by considering how levitating objects can be used as the interactive elements for static objects.

\subsection{Pervasive Displays from Physical Objects}

Our work builds on existing methods of augmenting static objects or materials, to turn them into dynamic displays and enable their use in new interactive contexts.

Projection is a common approach for adding dynamic output to ordinary surfaces and objects, turning them into rich displays by adding high resolution content. For example, the LightBeam [8] projected content onto objects in the user's surroundings (e.g., coffee mugs) and Funk et al. [6] created an interactive shower curtain using projection. Others have used drones to project onto the ground near users, for ad hoc displays for navigation $[3,11]$.

Lighting can similarly be manipulated to turn objects into simple displays. The ambientROOM [10] proposed the use of lighting to add dynamic output to objects and surfaces; for example, to change the appearance of a wall. Beams of light can also be used as interactive displays; for example, Tangible Lights [21] allowed users to interact with a spotlight by using their hands to cast shadows in the beam.

Others have developed low fidelity ambient displays by indirectly actuating objects and materials. For example, Plantxel [7] used airflow to actuate plant leaves, so that ordinary houseplants could be turned into meaningful ambient displays. Aireal [20] used air vortices to deliver haptic feedback over long distances $(\approx 3 \mathrm{~m})$ and suggested it could also be used to actuate objects in the environment, e.g., paper models and plant leaves. Pin wheels [9] similarly envisioned the use of air to manipulate small windmills, for ambient communication through the medium of airflow.

Our use of acoustic levitation complements these methods for turning ordinary objects and materials into interactive displays. Acoustic levitation allows particles to be precisely actuated around existing objects. The particles add a dynamic display component and can communicate meaningful information through their position and movements. Levitation also affords interactivity, because the particles can be used to give dynamic feedback about the user's interactions with the system.

\section{LEVITATION AROUND PHYSICAL OBJECTS}

We now explore how actuated levitating particles can be used to enhance physical objects and turn them into interactive displays. In this section, we describe the novel design possibilities afforded by the addition of levitating particles. We begin with a summary of the design parameters for presenting and manipulating particles. Then, we describe several ways of using particles to add interactivity through dynamic and responsive output. Later in the paper, we 
address the technical challenges of achieving acoustic levitation around physical objects.

\subsection{Levitating Particles}

A variety of materials can be levitated using acoustic elements or standing waves, so long as they satisfy two key constraints: their size does not exceed half of the sound wavelength and their density is low enough. Most levitating particles in HCI research are expanded polystyrene (EPS) beads $(\varnothing<5 \mathrm{~mm})$, because their low density means they levitate well. Others have recently levitated food and liquids as well [22]. Our designs use EPS beads for the particles because they are readily available and easy to levitate.

Levitating particles can be moved in any direction, at high speeds (e.g., up to $80 \mathrm{~cm} \mathrm{~s}^{-1}$ using standing wave levitation [1]). Multiple particles can be moved independently [18]. The maximum number of independently controlled particles depends on the number of ultrasound transducers and the strength of the sound field they produce [14]. A commonly used levitation system can dynamically manipulate 12 particles simultaneously [14], although our designs focus on smaller numbers. In our work, particles are not the main way of presenting content, so large numbers are not necessary.

\subsection{Using Particles to Enhance Physical Objects}

We identified three methods of using actuated levitating particles to create interactive displays from static physical objects. We arrived at these methods through a series of design exercises with artists, interaction designers and levitation experts, focused on exploring the combination of levitating beads with other items. The methods presented here show the key ways that particles were used in these designs. In this section, we describe how the particles were used to communicate information and create rich interactive experiences with other objects. We describe example systems that show these concepts, including implemented prototypes.

3.2.1 Particles for annotation. Particles can be used as dynamic cursors for annotating an object: e.g., to highlight points of interest, to draw a user's attention to a particular feature, or to add context to other sensory information. The particles annotate the object without affecting its appearance and without directly manipulating it. The annotation could be automated (e.g., a programmed series of movements to different locations) or interactive, responding to a user's input (e.g., "show me where that is"). A single particle can provide annotation by moving to different positions. Multiple particles could also be used, with subtle motion 'animations' to show the user which annotation is currently active.

For example, a museum exhibit could use a levitating particle to accompany playback of a narration, moving to interesting features of an artefact to indicate what the narrator is referring to. As an implemented example, we used a particle to show hiking routes across a mountain (Figure 2), with an interactive system that can visualise three routes. At the highest point in each visualisation, the particle paused whilst the system read the name of the summit and its altitude. We describe the implementation of this, and our other prototypes, later in the paper.

3.2.2 Particles for user representation. Particles can be used as the active user representation in an interactive system: e.g., as a cursor directly controlled by the user (like the LeviCursor [1]) or as a display element that gives feedback about the user's actions [4]. The particle turns a static object into an interactive display, by providing an effective way of representing the user and the effects of their interactions with the system.

For example, a user could explore a miniature model of a town by controlling the movements of a levitating particle. As the particle approaches landmarks in the model, the system could read their name and tourist information about them. Games could also be created from the skill required to control a levitating particle, e.g., by guiding one through a maze. We prototyped a golf game (Figure 1, middle), where users aimed a levitating particle (the golf ball) and it moved above the course, reacting to the terrain. Here, the particle provides a novel way of augmenting reality with gameplay.

3.2.3 Particles as animated display elements. Particles can be used to animate static objects, by providing a dynamic visual element to the content. This could be used to enhance the appearance of an object to create a more engaging experience, or to illustrate events or behaviours that are difficult to convey statically.

For example, levitating particles could be used as moving planets in a model of the Solar System, turning it into a dynamic display. We created a prototype that used levitating particles to show steam rising from the crater of a volcano (Figure 3). In this instance, the 'steam' is visually distinct from the volcano itself, held in place by ultrasound, rather than a physical attachment. We animated the steam particles with subtle shaking motions, to emphasise the active nature of this phenomena.

Animated particles can also be used visualise information that would not be apparent through an object's appearance. For example, they could be used to visualise the strength of the current in a river or gusts of wind, physical phenomena that would otherwise not be easily represented in static media. This enables objects to present richer information about their context.

3.2.4 Discussion of the design space. These methods use levitating particles to create dynamic displays out of static objects. Despite the small size and simplicity of the particles, they can convey a variety of information and enable a range of interactive experiences.

The information capacity of these simple particles is enhanced through the context in which they appear. When using a particle

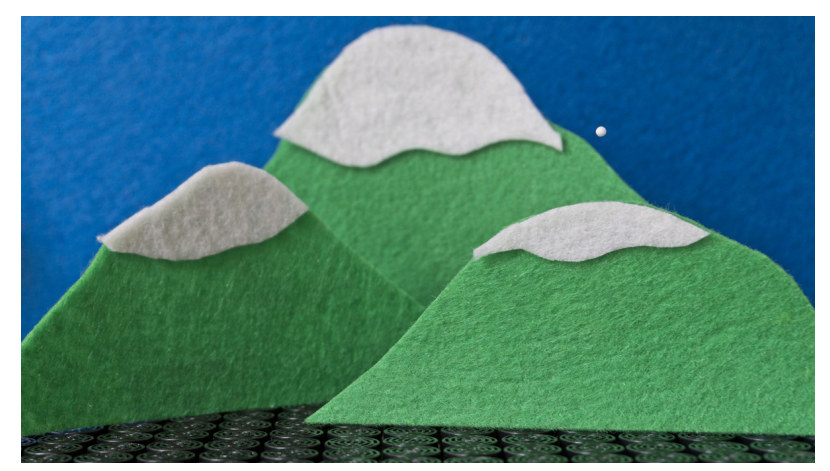

Figure 2: A model of a mountain range atop an ultrasound array, with a levitating bead that traces hiking routes. 


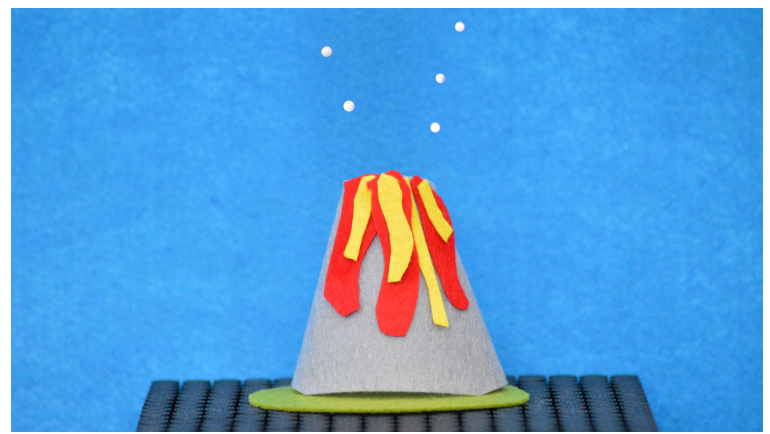

Figure 3: A volcano with 'steam' rising from its crater.

to annotate a physical object, its position relative to that object and its features is meaningful. Other levitation systems have used the positions of particles relative to each other to encode information (e.g., for comparing data points in Floating Charts [19]), but our designs exploit proximity to the nearby object instead.

When using a particle as the animated element in a display, the rest of the display content adds meaning to the particle's motions and behaviour. Individual levitating particles can be animated in a limited number of ways (e.g., 'shaking' them from side to side [4]), but the context allows this limited vocabulary of animations to be reused for different purposes. For example, a particle might shake to draw the user's attention to a location on a map, or because it is being blown by the wind in a landscape. These animations add a dynamic liveliness to the static models without modifying them.

Information capacity is further enhanced when the motions of a particle are linked to an action the user has performed. The action itself, and the user's expectations about its outcomes, provide meaning for the particle's behaviour. For example, a particle might move to a new position because the user has pressed a button; their expectations of a response from the system add meaning to the particle's movements at that time. Particle movements may also be controlled in real time (e.g., [1]), allowing complex continuous interactions with an immediately visible response. Reacting to a user's actions through particle behaviour is the key mechanism for turning static items into interactive displays.

Particles are levitated using ultrasound, a non-visible actuation mechanism. This invisibility can be used to great effect, by allowing the particles and their dynamic motions to be visually distinct from their nearby object. For example, in our volcano prototype (Figure 3), the particles are effective at conveying rising steam because they are not visibly connected to the volcano. This emphasises that they are a distinct entity. In our experience, this also increases engagement with our displays because onlookers are drawn in by the apparent 'magic' of levitation, as the particles levitate stably in air with no obvious actuation mechanism.

Many particles can be levitated and moved independently, to increase the scale of information communicated by these displays. This allows the presentation of multiple pieces of information; for example, adding several annotations to an object, or representing two users in a game. Multiple particles can also be used to create composite augmentations; for example, an animated display element using four particles in a straight line.
In this section, we explored how levitating particles can be used alongside static physical objects to create new interactive displays. The levitating particles may be simple in appearance, but create rich interactive experiences by the ways they are actuated next to other objects. We described ways of using particles as dynamic elements in this type of display and presented exemplary designs that demonstrate our ideas. In the next section, we address the technical challenges of realising these displays.

\section{METHODS FOR COMBINING LEVITATION WITH PHYSICAL OBJECTS}

There are many ways of allowing levitating particles to be actuated around physical objects. The naive method would be to place an acoustic levitator directly in front of the objects, but we look at alternatives that enable levitation in the space fully surrounding them instead. This is important because it allows more meaningful content presentation. It also offers greater visibility, since viewers do not need to look straight-on to see how the levitating particles relate to the physical objects in the background.

We explore three novel methods for adding actuated levitating particles to the space surrounding physical objects: placing a single ultrasound emitter above the objects; placing objects on top of a single-sided emitter or inside a double-sided emitter; and placing acoustically transparent physical objects below, on top, or inside an emitter. Figure 4 illustrates these object placements.

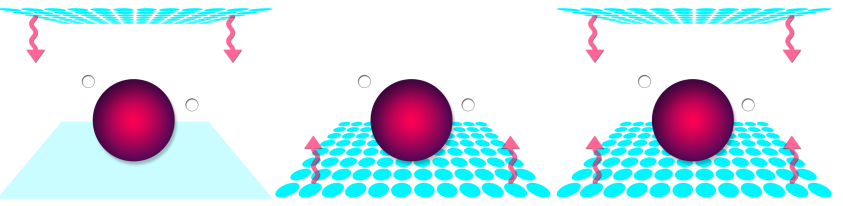

Figure 4: Physical objects can be put: (1) on a solid surface beneath an emitter; (2) on top of an emitter; or (3) inside a double-sided emitter. Arrows show ultrasound direction.

\subsection{Ultrasound Emitter Above an Object}

Our first method is to place a single ultrasound emitter above an object, so that ultrasound is directed towards the object and surface beneath it. The solid surface acts as an acoustic reflector, creating standing ultrasound waves for levitation (see Section 2.1). A focused standing wave will allow an object to be actuated in-air.

However, the physical object beneath the emitter will also reflect ultrasound, interfering with the standing waves. For this method to work in practice, the physical object will need to be considered when generating the ultrasound field for levitation. This needs to happen in real-time if users are able to manipulate or interact with the physical object, as any change in position or orientation will result in different ultrasound reflections.

Existing ultrasound field simulators (e.g., Ultraino [13], which we use) could be extended to incorporate the geometry of the physical object. Thus, when generating the control signals for the ultrasound emitters, the levitation standing waves will be generated taking the reflections into consideration. An implementation of this is out of the scope of this paper, but is an exciting area for future research. 
An advantage of this method is that it only requires a single ultrasound emitter, since the surface beneath the object reflects ultrasound to create standing waves. Visibility may be affected by the ultrasound array above the object, so would be most suitable for items that are displayed at eye-level. For example, an exhibit in a museum or gallery. Standing waves provide strong and reliable levitation, but this method needs to take reflections from the object into consideration. This may require real-time tracking if the physical object is likely to be moved by users.

\subsection{Objects Inside, or Above, an Emitter}

Our second method is to place a physical object inside a double sided levitator or on top of a single ultrasound emitter. If using a double sided emitter, the standing wave approach would be used. If using a single emitter, acoustic elements would be necessary for levitation. Like our first method, acoustic reflections from the physical objects would need to be taken into consideration when generating the ultrasound field for levitation.

Others have investigated levitation around a physical object placed on top of a single ultrasound emitter. SoundBender [16] is a method for bending ultrasound around an obstacle, so that reflections do not interfere with levitating objects. That method used acoustic metamaterials, physical structures placed on top of the ultrasound emitter that affect the propagation of the sound waves. Sound beams are effectively bent around the obstacles, allowing objects to be levitated in a 'twin trap' element (see Section 2.1), which can be actuated by manipulating the ultrasound from the emitter. In their paper, they demonstrate this with a levitating 'baseball' next to a Lego minifigure.

A key difference between this and the previous method is that objects are placed over an ultrasound emitter. For a single emitter, this offers the best possible visibility: the space surrounding the object is not occluded by an overhead device. However, objects may block some of the ultrasound transducers, potentially reducing the strength of the ultrasound field. Acoustic metamaterials (e.g., [16]) can mitigate this by redirecting the sound around the object instead.

A double-sided emitter for standing wave levitation may be more reliable than acoustic element levitation, since there can be twice the number of transducers and sound pressure comes from two directions, rather than one. This comes at a cost of reduced visibility (from the second emitter), but can allow a greater number of levitating particles. However, the effect of blocked transducers needs to be taken into account. This effect could be mitigated by raising an object off the surface of the lower emitter, or placing the emitters upright on each side of the object (e.g., Figure 5).

\subsection{Acoustically Transparent Objects Inside, Above, or Beneath an Emitter}

Our third method extends the previous two, using the concept of acoustic transparency to mitigate the impact of a physical object placed inside, on top of, or beneath an ultrasound emitter. An object or material is acoustically transparent if sound waves can pass through it. These are typically materials whose internal structure has small gaps $(10-100 \mu \mathrm{m})$ that sound can pass through. Since sound waves pass through them, they do not block or reflect ultrasound, allowing particles to be levitated as if no object was there.
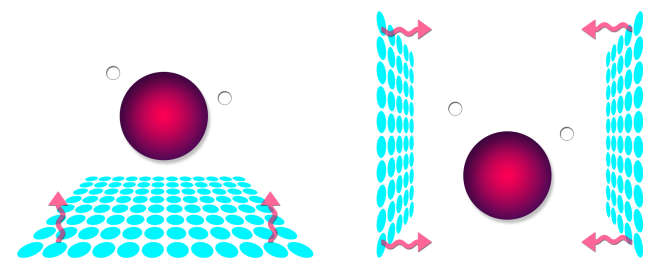

Figure 5: To avoid blocking transducers, objects could be raised from the lower emitter (left) or the emitters turned on their side (right), creating distance between the object and the transducers, for ultrasound to propagate.

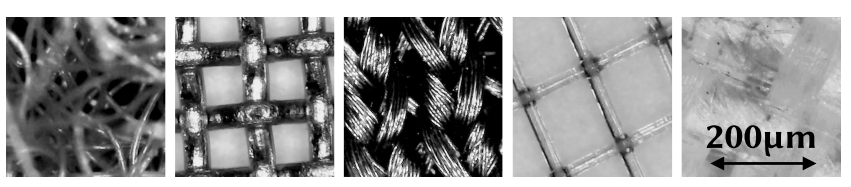

Figure 6: Microscopic images of materials that are partially transparent to $40 \mathrm{kHz}$ sound (left $\rightarrow$ right): acrylic felt, fine steel mesh, rayon, organza, polyester. The small gaps in the material structure allow sound to pass through.

Figure 6 shows examples of acoustically transparent materials. These include strong rigid materials (steel mesh), flexible materials that are visibly opaque (acrylic felt, rayon), translucent (organza), or can be projected onto (polyester). These materials can be used to construct physical objects; e.g., using steel mesh for structural support with other materials for external appearance.

Physical objects made from acoustically transparent materials can be placed inside, on top of, or below an ultrasound emitter. Ultrasound passes through the objects, allowing acoustic elements or standing waves to levitate particles nearby. Unlike the previous two methods, it is not necessary to consider the surface geometry of the object when generating the ultrasound field for levitation. This simplifies implementation, although tracking may be desirable for avoiding collisions between particles and the object. An additional benefit is that transparent objects do not block ultrasound from the emitters (i.e., when placed on top of the transducers), allowing a stronger sound field for better quality levitation.

This method is not compatible with existing objects, like museum artefacts, toys, or household ornaments, which are not likely to be fabricated from acoustically transparent materials. When adding levitation to such objects, the previous two approaches should be used instead. However, this approach is ideal when new objects can be created using suitable materials.

4.3.1 Investigating the transparency of materials. Whilst ultrasound can pass through acoustically transparent materials, their effect on an ultrasound wave are not fully understood. The structure of the materials may cause the ultrasound waves to bend, de-focusing a focal point slightly. To understand the effect of these materials on $40 \mathrm{kHz}$ ultrasound, we measured amplitude transmission through the five transparent materials in Figure 6. These measurements show how much amplitude passes through the materials, which has a direct impact on the reliability of acoustic levitation, as a stronger 'trap' is better able to hold a levitating object in air. 
To measure amplitude transmission, we recorded sound pressure levels with a receiving transducer, $15 \mathrm{~cm}$ above an emitting transducer. These were compared to a reference measurement (i.e., no material between them). We took measurements for 1-4 stacked layers of each material, since multiple layers are likely to be used when fabricating complex and detailed objects. Our measurements were conducted with the layers of materials placed flat across an ultrasound transducer, perpendicular to the direction of propagation. The effects of a material on sound waves will be influenced by its final shape and structure (e.g., a sphere vs. a cube). However, these measurements give an initial look at the degree of transparency of different materials and can inform material choice.

The results are shown in Table 1. Steel mesh was the second most transparent material. This is surprising as it is the strongest material we tested, but convenient because it provides strong structural support for 3D physical objects. Acrylic felt was the most opaque, passing only $77.6 \%$ amplitude through a single layer. We used felt in our prototypes for its aesthetic qualities, but our results show it is not ideal for designs with many layers (e.g., for detail or colour). In those cases, rayon (a natural woven fibre) may be more appropriate, as more ultrasound can pass through.

Table 1: Amplitude transmissivity of tested materials. Note: larger is better, i.e., more sound passes through.

\begin{tabular}{cccccc}
\hline Layers & $\begin{array}{c}\text { Acrylic } \\
\text { Felt }\end{array}$ & $\begin{array}{c}\text { Steel } \\
\text { Mesh }\end{array}$ & Rayon & Organza & Polyester \\
\hline 1 & $77.6 \%$ & $95.0 \%$ & $89.5 \%$ & $97.9 \%$ & $86.3 \%$ \\
2 & $59.7 \%$ & $93.7 \%$ & $82.1 \%$ & $96.0 \%$ & $67.4 \%$ \\
3 & $45.0 \%$ & $88.7 \%$ & $77.6 \%$ & $94.7 \%$ & $51.3 \%$ \\
4 & $33.4 \%$ & $84.2 \%$ & $66.1 \%$ & $93.7 \%$ & $41.6 \%$ \\
\hline
\end{tabular}

4.3.2 Fabricating acoustically transparent objects. To demonstrate the feasibility of this concept, we made a variety of physical objects from acoustically transparent materials. These include a model of a mountain range (Figure 2), a hole on a golf course and a volcano (Figure 3). Earlier in this paper, we described the ways we used levitating particles to add dynamic display components and interactive elements to these models. We now describe how we created them, integrated them with ultrasound devices, and implemented the dynamic particle behaviours.

The mountains were created from acrylic felt, held upright by steel mesh supports. Layers of felt were used to add detail (the snow on the summits) and were joined together, and to the steel mesh, using thread. The extremely fine diameter of the thread has minimal impact on the ultrasound waves, as they simply bend around it. Figure 1 shows the mountains placed directly on top of a single ultrasound emitter (a 16x16 $40 \mathrm{kHz}$ transducer array). An EPS bead, levitated in a twin trap [15], moved to show hiking routes.

The golf course was created using several layers of acrylic felt and was placed directly over the bottom array of transducers in a double sided ultrasound emitter. The layers were held together using thread, as before. Our results from the earlier measurements suggest that several layers of felt will reduce the amplitude passed through the golf course, so we used a double-sided emitter to give strong levitation in this instance (two $16 \times 1640 \mathrm{kHz}$ arrays, held $23 \mathrm{~cm}$ apart). Because the model only made sparse use of multiple layers, the ultrasound was strong enough to allow a levitating EPS bead (i.e., golf ball) to be moved quickly above it, without falling out of its standing wave trap. We altered the trajectory of the particle as it moved, rising over the hills and falling through the bumps.

The volcano was created using acrylic felt, wrapped around a steel support structure to help it hold its shape. The volcano was placed inside the double-sided emitter described before. The 'steam' particles above the crater were animated to gently move from side to side, creating a subtle appearance of motion.

Ultrasound arrays are becoming increasingly accessible, both commercially and through self-build guides with low-cost components [2, 12]. Our hardware (described in [14]) was driven using Ultraino [13], an open-source framework for acoustic levitation. We used its implementation of the ITR algorithm (with 5 iterations) to create levitation points, as this is reliable and runs in real-time. Frameworks like this help designers overcome technical barriers to levitation and do not require much computational resources, performing well on a standard laptop computer.

\section{CONCLUSION}

We explored the use of acoustic levitation to create novel dynamic displays from static physical objects. We presented three ways of using actuated particles to create new interactive experiences with these static objects: by annotating and drawing attention to their features; by adding an active user representation for interactivity; and by creating dynamic display components that enhance their appearance. Whilst the particles are simple in appearance, their proximity to other objects and their features allow them to communicate rich information through their motions. Expressiveness is further enhanced when the motions are linked to a user's actions.

We described three methods for actuating levitating particles around physical objects. Two involved placing objects on or inside an acoustic levitation device. These can create pervasive displays from objects that cannot be modified or directly interacted with; for example, actuated particles could be used to add interactivity to museum artefacts or artworks in a gallery, without modifying or damaging them. Our third method used acoustically transparent materials for fabrication, so that objects can be placed inside a levitator, without significantly disrupting the ultrasound. We looked at the effects of five materials on ultrasound. Our results and physical prototypes show the feasibility of fabricating objects from acoustically transparent materials.

With this work, we investigated a new application of acoustic levitation. Instead of using small levitating particles as the sole means of presenting content, we envision their use as actuated components for other objects. Levitation has the potential to enable new interactions with other objects and displays, and we hope to encourage others to think about similar ways of using levitating particles for new interactive experiences.

\section{ACKNOWLEDGEMENTS}

This work is funded by the European Union H2020 research and innovation programme (\#737087: Levitate) and is supported by University of Bristol and Universidad Publica de Navarra. 


\section{REFERENCES}

[1] Myroslav Bachynskyi, Viktorija Paneva, and Jörg Müller. 2018. LeviCursor: Dexterous Interaction with a Levitating Object. In Proceedings of the 2018 ACM International Conference on Interactive Surfaces and Spaces - ISS '18. ACM Press, 253-262. https://doi.org/10.1145/3279778.3279802

[2] William Beasley, Brenda Gatusch, Daniel Connolly-Taylor, Chenyuan Teng, Asier Marzo, and Jose Nunez-Yanez. 2019. High-Performance Ultrasonic Levitation with FPGA-based Phased Arrays. (2019). arXiv:1901.07317 http://arxiv.org/abs/ 1901.07317

[3] Anke M. Brock, Julia Chatain, Michelle Park, Tommy Fang, Martin Hachet, James A. Landay, and Jessica R. Cauchard. 2018. FlyMap: Interacting with Maps Projected from a Drone. In Proceedings of the 7th ACM International Symposium on Pervasive Displays - PerDis '18. ACM Press, Article 13. https://doi.org/10.1145/ 3205873.3205877

[4] Euan Freeman, Julie Williamson, Sriram Subramanian, and Stephen Brewster. 2018. Point-and-Shake: Selecting from Levitating Object Displays. In Proceedings of the 36th Annual ACM Conference on Human Factors in Computing Systems CHI '18. ACM Press, Paper 18. https://doi.org/10.1145/3173574.3173592

[5] Euan Freeman, Julie R. Williamson, Praxitelis Kourtelos, and Stephen Brewster 2018. Levitating Particle Displays with Interactive Voxels. In Proceedings of the 7th ACM International Symposium on Pervasive Displays - PerDis '18. ACM Press.

[6] Markus Funk, Stefan Schneegass, Michael Behringer, Niels Henze, and Albrecht Schmidt. 2015. An Interactive Curtain for Media Usage in the Shower. In Proceedings of the 4th International Symposium on Pervasive Displays - PerDis '15. ACM Press, 225-231. https://doi.org/10.1145/2757710.2757713

[7] Vito Gentile, Salvatore Sorce, Ivan Elhart, and Fabrizio Milazzo. 2018. Plantxel: Towards a Plant-based Controllable Display. In Proceedings of the 7th ACM International Symposium on Pervasive Displays PerDis '18. ACM Press, Article 16. https://doi.org/10.1145/3205873.3205888

[8] Jochen Huber, Jürgen Steimle, Chunyuan Liao, Qiong Liu, and Max Mühlhäuser 2012. LightBeam: Interacting with Augmented Real-World Objects in Pico Projections. In Proceedings of the 11th International Conference on Mobile and Ubiquitous Multimedia - MUM '12. ACM Press, Article 16. https://doi.org/10.1145/2406367. 2406388

[9] Hiroshi Ishii, Sandia Ren, and Phil Frei. 2001. Pinwheels: Visualizing Information Flow in an Architectural Space. In Extended Abstracts of the SIGCHI Conference on Human Factors in Computing Systems - CHI '01. ACM Press, 111-112. https //doi.org/10.1145/634067.634135

[10] Hiroshi Ishii, Craig Wisneski, Scott Brave, Andrew Dahley, Matt Gorbet, Brygg Ullmer, and Paul Yarin. 1998. ambientROOM: Integrating Ambient Media with Architectural Space. In Proceedings of the SIGCHI Conference on Human Factors in Computing Systems - CHI '98. ACM Press, 173-174. https://doi.org/10.1145/ 286498.286652
[11] Pascal Knierim, Steffen Maurer, Katrin Wolf, and Markus Funk. 2018. QuadcopterProjected In-Situ Navigation Cues for Improved Location Awareness. In Proceedings of the 2018 CHI Conference on Human Factors in Computing Systems - CHI '18. ACM Press, Paper 433. https://doi.org/10.1145/3173574.3174007

[12] Asier Marzo. 2018. Ultrasonic Array Instructables. http://www.acousticlevitator. com/

[13] Asier Marzo, Tom Corkett, and Bruce W. Drinkwater. 2018. Ultraino: An Open Phased-Array System for Narrowband Airborne Ultrasound Transmission. IEEE Transactions on Ultrasonics, Ferroelectrics, and Frequency Control 65, 1 (2018), 102-111. https://doi.org/10.1109/TUFFC.2017.2769399

[14] Asier Marzo and Bruce W. Drinkwater. 2019. Holographic acoustic tweezers. Proceedings of the National Academy of Sciences 116, 1 (2019), 84-89. https: //doi.org/10.1073/pnas.1813047115

[15] Asier Marzo, Sue Ann Seah, Bruce W. Drinkwater, Deepak Ranjan Sahoo, Benjamin Long, and Sriram Subramanian. 2015. Holographic acoustic elements for manipulation of levitated objects. Nature Communications 6 (2015), Article 8661. https://doi.org/10.1038/ncomms9661

[16] Mohd Adili Norasikin, Diego Martinez Plasencia, Spyros Polychronopoulos, Gianluca Memoli, Yutaka Tokuda, and Sriram Subramanian. 2018. SoundBender: Dynamic Acoustic Control Behind Obstacles. In Proceedings of the 31st ACM User Interface Software and Technology Symposium - UIST '18. ACM Press, 247-259. https://doi.org/10.1145/3242587.3242590

[17] Yoichi Ochiai, Takayuki Hoshi, and Jun Rekimoto. 2014. Pixie Dust: Graphics Generated by Levitated and Animated Objects in Computation Acoustic-Potential Field. ACM Transactions on Graphics 33, 4 (2014), Article 85. https://doi.org/10. $1145 / 2601097.2601118$

[18] Themis Omirou, Asier Marzo, Sue Ann Seah, and Sriram Subramanian. 2015. LeviPath: Modular Acoustic Levitation for 3D Path Visualisations. In Proceedings of the 33rd Annual ACM Conference on Human Factors in Computing Systems CHI '15. ACM Press, 309-312. https://doi.org/10.1145/2702123.2702333

[19] Themis Omirou, Asier Marzo Perez, Sriram Subramanian, and Anne Roudaut. 2016. Floating Charts: Data Plotting using Free-Floating Acoustically Levitated Representations. In 2016 IEEE Symposium on 3D User Interfaces (3DUI). IEEE, 187-190. https://doi.org/10.1109/3DUI.2016.7460051

[20] Rajinder Sodhi, Ivan Poupyrev, Matthew Glisson, and Ali Israr. 2013. AIREAL: Interactive Tactile Experiences in Free Air. ACM Transactions on Graphics 32, 4 (2013), Article 134. https://doi.org/10.1145/2461912.2462007

[21] Tor Sørensen, Oskar D. Andersen, and Timothy Merritt. 2015. "Tangible Lights": In-Air Gestural Control of Home Lighting. In Proceedings of the 9th International Conference on Tangible, Embedded, and Embodied Interaction - TEI'15 Work-inProgress. ACM Press, 727-732. https://doi.org/10.1145/2677199.2687909

[22] Chi Than Vi, Asier Marzo, Damien Ablart, Gianluca Memoli, Sriram Subramanian, Bruce Drinkwater, and Marianna Obrist. 2017. TastyFloats: A Contactless Food Delivery System. In Proceedings of the 2017 ACM International Conference on Interactive Surfaces and Spaces - ISS '17. ACM Press, 161-170. https://doi.org/10. $1145 / 3132272.3134123$ 